\title{
AVALIAÇÃO DO PERFIL LIPÍDICO EM ADULTOS NO SUL DO BRASIL
}

\author{
LIPID PROFILE IN ADULTS FROM WEST FRONTIER \\ OF RIO GRANDE DO SUL, BRAZIL
}

\section{Carolina Simões Pires Ribeiro', Beatris Gonzalez Cademartori², Andréa da Silva Ramos Rocha ${ }^{3}$}

\section{RESUMO}

Objetivo: avaliar o perfil lipídico em adultos de um laboratório de análises clínicas em Santana do Livramento/RS. Metodologia: estudo transversal retrospectivo quantitativo desenvolvido em um laboratório com 944 indivíduos que realizaram dosagens do perfil lipídico. Resultados: a maioria dos indivíduos apresentou dosagens de lipídeos fora do desejável. Foi observada diferença estatisticamente significante entre os sexos e os valores de HDL-c $(p \leq 0,001)$ e triglicerídeos ( $p \leq$ $0,001)$. Associação significativa foi observada entre a faixa etária e as dosagens de colesterol total $(p \leq 0,001)$ e LDL-c $(p=0,010)$, para ambos os sexos. Percentuais elevados de hipercolesterolemia, hipertrigliceridemia isolada, hiperlipidemia mista e HDL-c baixo foram observados em indivíduos a partir dos 40 anos de idade. Conclusão: os resultados encontrados evidenciaram uma forte tendência ao desenvolvimento de doenças cardiovasculares a partir de 40 anos de idade e poderão contribuir para um planejamento preventivo de estratégias de saúde pública na região.

Descritores: Prevalência; Dislipidemias; Lipídeos
${ }^{1}$ Mestre em Ciências - Bioquímica pela Universidade Federal do Paraná (UFPR), Curitiba, PR, Brasil.

2 Doutora em Parasitologia pela Universidade Federal de Pelotas (UFPEL), Pelotas, RS, Brasil.

${ }^{3}$ Doutora em Biotecnologia pela Universidade Federal de Pelota (UFPEL), Pelotas, RS, Brasil.

\begin{abstract}
The objective of this study was to evaluate the lipid profile in adults of a clinical analysis laboratory in Santana do Livramento- RS. Retrospective cross-sectional study developed in a laboratory with 944 those subjects who performed dosages of the lipid profile. Most subjects had lipid dosages outside desirable. There was a statistically significant difference between the sexes and HDL-C values $(p \leq 0.001)$ and triglycerides $(p \leq 0.001)$. A significant association was observed between age and total cholesterol $(p \leq 0,001)$ and LDL-C $(p=0.010)$ for both sexes. Percentuais elevados de hipercolesterolemia, hipertrigliceridemia isolada, hiperlipidemia mista e HDL-c baixo foram observados em indivíduos a partir dos 40 anos de idade. The results evidence a strong tendency to develop cardiovascular disease from 40 years of age and may contribute to a preventive planning public health strategies in the region.
\end{abstract}

Descriptors: Prevalence; Dyslipidemias; Lipids 


\section{Introdução}

As dislipidemias são caracterizadas por distúrbios no metabolismo dos lipídeos. Estão diretamente relacionadas ao fator de risco de desenvolvimento de doenças cardiovasculares (DCV), sendo esta uma das principais causas de mortalidade em adultos ${ }^{1}$. Em busca da redução da incidência dessas doenças, têm-se estudado a sua fisiopatologia e identificado fatores de risco ligados ao seu desenvolvimento ${ }^{2,3}$.

Vários são os fatores de risco para o aparecimento de DCV, como sedentarismo, hábito alimentar inadequado, tabagismo, etilismo e histórico familiar de hiperlipidemia ${ }^{4}$. De acordo com a Sociedade Brasileira de Cardiologia, 0 perfil lipídico deve ser realizado para controlar o aparecimento ou para o monitoramento de suas complicações. Este é determinado pelas dosagens bioquímicas de colesterol total, HDL colesterol, LDL colesterol e triglicerídeos 5 .

Níveis elevados de colesterol total, LDL colesterol e triglicerídeos, assim como níveis reduzidos de HDL colesterol estão relacionados com maior incidência de hipertensão e doença aterosclerótica ${ }^{6}$.

Com progressão lenta, a aterosclerose se inicia na infância sendo caracterizada pela formação de ateromas (depósitos de lipídeos na camada íntima das artérias) que provocam a restrição do fluxo sanguíneo. 0 estreitamento da luz do vaso pode levar à obstrução e surgimento de suas manifestações clínicas, como infarto do miocárdio, acidente vascular cerebral e doença vascular periférica ${ }^{7}$.

A prevalência das dislipidemias em uma região pode variar segundo características socioeconômicas, sexo, idade, estilo de vida e hábitos dietéticos culturais ${ }^{8}$. A fronteira oeste do Rio Grande do Sul é uma região caracterizada pelo elevado consumo de alimentos gordurosos por influência cultural e, por este ser um dos fatores relacionado ao desenvolvimento das dislipidemias, a população desta região poderá estar mais suscetível ao desenvolvimento desta enfermidade.

Sendo assim, este estudo teve como objetivo avaliar o perfil lipídico de indivíduos adultos de um laboratório de análises clínicas, no município de Santana do Livramento, fronteira oeste do Rio Grande do Sul, Brasil.

\section{Metodologia}

Um estudo transversal retrospectivo qualitativo foi realizado, no período de janeiro de 2012 a janeiro de 2013, em um laboratório de análises clínicas, localizado na cidade de Santana do Livramento, fronteira oeste do estado do Rio Grande do Sul. A pesquisa foi aprovada pelo Comitê de Ética em Pesquisa em Seres Humanos da Universidade Católica de Pelotas sob o Protocolo n 377.733 /Julho 2013.

As amostras foram selecionadas a partir do banco de dados do laboratório. Foram selecionados todos os indivíduos com idade igual ou superior a 20 anos, que realizaram exames para avaliar o perfil lipídico durante o período do estudo. No total foram avaliados 944 indivíduos, de ambos os sexos, com idade de 20 a 59 anos. Foram excluídos do estudo, aqueles que realizaram exames de repetição (monitoramento terapêutico ou confirmação de resultados anteriores) nesse intervalo de tempo.

As dosagens séricas de colesterol total (CT), triglicerídeos (TG) e colesterol HDL (HDL-c) foram realizadas por método colorimétrico enzimático no equipamento automatizado Integra 400 (Roche $\left.{ }^{\circledR}\right)$. 0 colesterol LDL (LDL-c) foi obtido através de cálculo de Friedewald para triglicerídeos abaixo de 400mg/dL: LDL=CT-(HDL-c+TG/5)9 .

Para a avaliação do perfil lipídico e classificação das dislipidemias foram adotados os valores referenciais estabelecidos pela V Diretriz Brasileira sobre Dislipidemias e Prevenção da Aterosclerose (V DBPA) para indivíduos com idade a partir de 20 anos $^{5}$.

Posteriormente, todos os indivíduos que apresentaram alteração nas frações lipídicas foram incluídos na classificação das dislipidemias, em quatro tipos: hipercolesterolemia isolada, hipertrigliceridemia isolada, hiperlipidemia mista e HDL-c baixo (Tabela 1). 
Tabela 1 - Classificação das dislipidemias segundo a

V Diretriz Brasileira de Dislipidemia e Prevenção da Aterosclerose.

\begin{tabular}{c|c}
\hline Hipercolesterolemia isolada & $\mathrm{LDL}-\mathrm{c} \geq 160 \mathrm{mg} / \mathrm{dL}$ \\
\hline Hipertrigliceridemia isolada & $\mathrm{TC} \geq 150 \mathrm{mg} / \mathrm{dL}$ \\
\hline Hiperlipidemia mista & $\mathrm{LDL}-\mathrm{c} \geq 160 \mathrm{mg} / \mathrm{dL}$ \\
$\mathrm{TC} \geq 150 \mathrm{mg} / \mathrm{dL}$ \\
\hline HDL-c baixo & $\mathrm{HDL}-\mathrm{c}<40 \mathrm{mg} / \mathrm{dL}$ (Homens) \\
& $\mathrm{HDL}-\mathrm{c}<50 \mathrm{mg} / \mathrm{dL}$ (Mulheres) \\
\hline
\end{tabular}

\section{Análise estatística}

Os dados foram validados por dupla digitação e análise estatística realizada pelo Programa Stata versão 12.0 (Stata Corporation, College Station, TX, USA). A análise descritiva foi realizada para descrever as frequências absolutas e relativas. Os testes Qui-Quadrado e Exato de Fisher foram realizados para analisar a associação entre as variáveis independentes e de interesse. Foi adotado um percentual de $95 \%$ de confiança $(p<0,05)$.

\section{Resultados}

Foram avaliados os exames laboratoriais de 944 indivíduos, sendo 551 (58,4\%) do sexo feminino. A média de idade foi 43,6 anos para mulheres e 45,2 anos para os homens.

Verificou-se que 78,1\% (737/944) dos indivíduos apresentaram dosagens de parâmetros do perfil lipídico fora do desejável segundo a V DBPA. No sexo feminino, os valores de colesterol total variaram de 205 a $559 \mathrm{mg} / \mathrm{dL}$, triglicerídeos de 150 a $3184 \mathrm{mg} / \mathrm{dL}, \mathrm{HDL}-\mathrm{c}$ de 21 a $59 \mathrm{mg} / \mathrm{dL}$ e, LDL-c de 130 a $256 \mathrm{mg} / \mathrm{dL}$. No sexo masculino, as taxas de colesterol total foram de 201 a $431 \mathrm{mg} / \mathrm{dL}$, triglicerídeos de 17 a $873 \mathrm{mg} / \mathrm{dL}$, HDL-c de 26 a $59 \mathrm{mg} / \mathrm{dL}$ e, LDL-c de 143 a $339 \mathrm{mg} / \mathrm{dL}$.

0 perfil lipídico dos participantes de acordo com o gênero, usando como critério a V Diretriz Brasileira Sobre Dislipidemias e Prevenção da Aterosclerose está apresentado na tabela 2. Os valores de HDL-c $(p \leq 0,001)$ e triglicerídeos ( $p \leq 0,001)$ quando comparados entre os gêneros apresentaram diferença estatisticamente significante. As mulheres apresentaram maiores percentuais nas categorias desejável $(81,1 \%)$ e limítrofe $(57,6 \%)$ de HDL-c em comparação aos homens. 0 percentual de homens foi maior em relação ao $\mathrm{HDL}-\mathrm{c}$ baixo $(60,1 \%)$. Em relação aos triglicerídeos, os maiores percentuais de valores alto e muito alto foram observados nos homens e, nas mulheres os maiores valores foram na categoria de desejável $(90,5 \%)$ e limítrofe $(54,4 \%)$. Não foi possível a realização da análise do LDL-c de 33 indivíduos, sendo 26 homens e 7 mulheres, pois apresentaram valores de triglicerídeos superiores a 400 $\mathrm{mg} / \mathrm{dL}$, o que impossibilita o cálculo pela equação de Friedewald, totalizando 911 indivíduos.

Tabela 2 - Resultados da avaliação do perfil lipídico dos pacientes, segundo o gênero, Santana do Livramento, RS ( $n=944)$.

\begin{tabular}{|c|c|c|c|c|c|c|c|}
\hline Lipídeos & \multirow{2}{*}{$\begin{array}{c}{ }^{* * *} \text { Categoria } \\
\mathbf{~ m g / d L}\end{array}$} & \multicolumn{2}{|c|}{ Masculino } & \multicolumn{2}{|c|}{ Feminino } & \multirow{2}{*}{$\begin{array}{c}\text { Total } \\
421\end{array}$} & $\begin{array}{c}\text { Valor de } \\
\qquad \text { p* }^{*}\end{array}$ \\
\hline \multirow{3}{*}{ CT } & & 158 & 37,5 & 263 & 62,5 & & \multirow{3}{*}{0,070} \\
\hline & Limítrofe 200-239 & 147 & 45,4 & 177 & 54,6 & 324 & \\
\hline & Alto $\geq 240$ & 88 & 44,2 & 111 & 55,8 & 199 & \\
\hline \multirow{4}{*}{ HDL-C } & Desejável >60 & 39 & 18,9 & 167 & 81,1 & 206 & \multirow{4}{*}{$\leq 0,001$} \\
\hline & Limítrofe 40-60 & 214 & 42,4 & 291 & 57,6 & 505 & \\
\hline & Baixo $<40$ & 140 & 60,1 & 93 & 39,1 & 233 & \\
\hline & Ótimo <100 & 69 & 34,7 & 130 & 65,3 & 199 & \\
\hline
\end{tabular}




\begin{tabular}{|c|c|c|c|c|c|c|c|}
\hline Lipídeos & $\begin{array}{c}{ }^{* * *} \text { Categoria } \\
\text { mg/dL }\end{array}$ & $\begin{array}{c}\text { Mas } \\
\mathbf{N}\end{array}$ & $\begin{array}{c}\text { ino } \\
\%\end{array}$ & $\begin{array}{r}\text { Fem } \\
\mathbf{N}\end{array}$ & $\begin{array}{r}\text { ino } \\
\%\end{array}$ & Total & $\begin{array}{c}\text { Valor de } \\
\text { p* }^{*}\end{array}$ \\
\hline \multirow{3}{*}{ LDL-C ${ }^{* * * *}$} & Desejável 100-129 & 109 & 37,5 & 182 & 62,5 & 291 & \multirow{3}{*}{0,062} \\
\hline & Limítrofe 130-159 & 121 & 44,5 & 151 & 55,5 & 272 & \\
\hline & Alto $\geq 160$ & 68 & 45,6 & 81 & 54,4 & 149 & \\
\hline \multirow{4}{*}{ TG** $^{* *}$} & Desejável <150 & 212 & 35,8 & 381 & 64,2 & 593 & \multirow{4}{*}{$\leq 0,001$} \\
\hline & Limítrofe 150-199 & 70 & 43,5 & 91 & 56,5 & 161 & \\
\hline & Alto $200-499$ & 92 & 54,4 & 77 & 45,6 & 169 & \\
\hline & Muito alto $\geq 500$ & 19 & 90,5 & 2 & 9,5 & 21 & \\
\hline
\end{tabular}

* Valores estatisticamente significantes com $\mathrm{p}<0,05 .{ }^{* *}$ Teste Exato de Fisher.

${ }^{* * *} \bigvee$ Diretriz Brasileira sobre Dislipidemias e Prevenção de Aterosclerose (2013). ${ }^{* * *} \mathrm{n}=911$

A tabela 3 apresenta o perfil lipídico segundo a faixa etária estratificada por gênero. No grupo dos homens foram encontradas diferenças significativas nos valores de colesterol total $(p \leq 0,001)$ e LDL-c $(p=0,010)$ quando comparado entre as faixas etárias. Foi observado que $81,1 \%$ dos homens com valores considerados altos ( $\geq 240 \mathrm{mg} / \mathrm{dL}$ ) para 0 colesterol total tinham idade a partir de 40 anos. Nesta mesma faixa etária, 80,9\% dos homens apresentaram valores altos ( $\geq 160 \mathrm{mg} / \mathrm{dL}$ ) para o LDL-c.

Para as mulheres, a análise estatística também mostrou diferença significativa entre as faixas etárias para 0 colesterol total $(p \leq 0,001)$ e LDL-c $(p \leq 0,001)$. Valores altos para colesterol total $(80,9 \%)$ e LDL-c $(79 \%)$ foram observados a partir de 40 anos de idade.

Tabela 3 - Avaliação do perfil lipídico dos pacientes, segundo gênero e faixa etária, Santana do Livramento, RS (n=944).

\begin{tabular}{|c|c|c|c|c|c|c|}
\hline \multirow[b]{2}{*}{ Lipídeos } & \multicolumn{3}{|c|}{ Masculino } & \multicolumn{3}{|c|}{ Feminino } \\
\hline & $20-29$ & $\begin{array}{l}\mathbf{N}(\%) \\
30-39\end{array}$ & $\geq 40$ & $20-29$ & $\begin{array}{l}\mathbf{N}(\%) \\
30-39\end{array}$ & $\geq 40$ \\
\hline \multicolumn{7}{|l|}{ TG** } \\
\hline $\begin{array}{c}\text { Desejável } \\
(<150 \mathrm{mg} / \mathrm{dL}) \\
\end{array}$ & $\begin{array}{c}25 \\
(11,8) \\
\end{array}$ & $\begin{array}{c}51 \\
(24,1) \\
\end{array}$ & $\begin{array}{c}136 \\
(64,1) \\
\end{array}$ & $\begin{array}{c}64 \\
(16,8) \\
\end{array}$ & $\begin{array}{c}79 \\
(20,7) \\
\end{array}$ & $\begin{array}{c}238 \\
(62,5) \\
\end{array}$ \\
\hline $\begin{array}{c}\text { Limítrofe } \\
\text { (150-199 mg/dL) }\end{array}$ & $\begin{array}{c}4 \\
(5,8)\end{array}$ & $\begin{array}{c}12 \\
(17,1) \\
\end{array}$ & $\begin{array}{c}54 \\
(77,1) \\
\end{array}$ & $\begin{array}{c}11 \\
(12,1) \\
\end{array}$ & $\begin{array}{c}16 \\
(17,6) \\
\end{array}$ & $\begin{array}{c}64 \\
(70,3)\end{array}$ \\
\hline $\begin{array}{c}\text { Alto } \\
(200-499 \mathrm{mg} / \mathrm{dL})\end{array}$ & $\begin{array}{c}5 \\
(5,4) \\
\end{array}$ & $\begin{array}{c}14 \\
(15,2) \\
\end{array}$ & $\begin{array}{c}73 \\
(79,4) \\
\end{array}$ & $\begin{array}{c}6 \\
(7,8) \\
\end{array}$ & $\begin{array}{c}12 \\
(15,6) \\
\end{array}$ & $\begin{array}{c}59 \\
(76,6) \\
\end{array}$ \\
\hline $\begin{array}{c}\text { Muito alto } \\
(\geq 500 \mathrm{mg} / \mathrm{dL})\end{array}$ & $\begin{array}{c}0 \\
(-)\end{array}$ & $\begin{array}{c}3 \\
(15,8)\end{array}$ & $\begin{array}{c}16 \\
(84,2)\end{array}$ & $\begin{array}{l}0 \\
(-)\end{array}$ & $\begin{array}{c}1 \\
(50)\end{array}$ & $\begin{array}{c}1 \\
(50)\end{array}$ \\
\hline Valor de p* & \multicolumn{3}{|c|}{0,058} & \multicolumn{3}{|c|}{0,188} \\
\hline \multicolumn{7}{|l|}{$\mathrm{CT}^{* *}$} \\
\hline $\begin{array}{c}\text { Desejável } \\
(<200 \mathrm{mg} / \mathrm{dL})\end{array}$ & $\begin{array}{c}24 \\
(15,2) \\
\end{array}$ & $\begin{array}{c}39 \\
(24,7)\end{array}$ & $\begin{array}{c}95 \\
(60,1) \\
\end{array}$ & $\begin{array}{c}62 \\
(23,6) \\
\end{array}$ & $\begin{array}{c}64 \\
(24,3) \\
\end{array}$ & $\begin{array}{c}137 \\
(52,1)\end{array}$ \\
\hline $\begin{array}{c}\text { Limítrofe } \\
(200-239 \mathrm{mg} / \mathrm{dL})\end{array}$ & $\begin{array}{c}6 \\
(4,1)\end{array}$ & $\begin{array}{c}29 \\
(19,7)\end{array}$ & $\begin{array}{c}112 \\
(76,2)\end{array}$ & $\begin{array}{c}11 \\
(6,2)\end{array}$ & $\begin{array}{l}30 \\
(17)\end{array}$ & $\begin{array}{c}136 \\
(76,8)\end{array}$ \\
\hline $\begin{array}{c}\text { Alto } \\
(\geq 240 \mathrm{mg} / \mathrm{dL})\end{array}$ & $\begin{array}{c}4 \\
(4,6)\end{array}$ & $\begin{array}{c}12 \\
(13,6)\end{array}$ & $\begin{array}{c}72 \\
(81,8)\end{array}$ & $\begin{array}{c}8 \\
(7,2)\end{array}$ & $\begin{array}{c}14 \\
(12,6)\end{array}$ & $\begin{array}{c}89 \\
(80,2)\end{array}$ \\
\hline Valor de p* & \multicolumn{3}{|c|}{$\leq 0,001$} & \multicolumn{3}{|c|}{$\leq 0,001$} \\
\hline
\end{tabular}




\begin{tabular}{|c|c|c|c|c|c|c|}
\hline \multirow[b]{2}{*}{ Lipídeos } & \multicolumn{3}{|c|}{ Masculino } & \multicolumn{3}{|c|}{ Feminino } \\
\hline & $20-29$ & $\begin{array}{l}\mathbf{N}(\%) \\
30-39\end{array}$ & $\geq 40$ & $20-29$ & $\begin{array}{l}\mathbf{N}(\%) \\
30-39\end{array}$ & $\geq 40$ \\
\hline \multicolumn{7}{|l|}{ HDL -c** } \\
\hline $\begin{array}{c}\text { Desejável } \\
\text { (> } 60 \text { mg/dL) }\end{array}$ & $\begin{array}{c}6 \\
(15,4) \\
\end{array}$ & $\begin{array}{c}5 \\
(12,8) \\
\end{array}$ & $\begin{array}{c}28 \\
(71,8) \\
\end{array}$ & $\begin{array}{c}26 \\
(15,6) \\
\end{array}$ & $\begin{array}{c}29 \\
(17,3) \\
\end{array}$ & $\begin{array}{c}112 \\
(67,1) \\
\end{array}$ \\
\hline $\begin{array}{c}\text { Limítrofe } \\
\text { (40- } 60 \text { mg/dL) }\end{array}$ & $\begin{array}{c}13 \\
(6,1) \\
\end{array}$ & $\begin{array}{r}45 \\
(21) \\
\end{array}$ & $\begin{array}{c}156 \\
(72,9) \\
\end{array}$ & $\begin{array}{c}42 \\
(14,4) \\
\end{array}$ & $\begin{array}{l}58 \\
(20) \\
\end{array}$ & $\begin{array}{c}191 \\
(65,6) \\
\end{array}$ \\
\hline $\begin{array}{c}\text { Baixo } \\
(<40 \mathrm{mg} / \mathrm{dL})\end{array}$ & $\begin{array}{c}15 \\
(10,7) \\
\end{array}$ & $\begin{array}{c}30 \\
(21,4) \\
\end{array}$ & $\begin{array}{c}95 \\
(67,9) \\
\end{array}$ & $\begin{array}{c}13 \\
(14) \\
\end{array}$ & $\begin{array}{c}21 \\
(22,6) \\
\end{array}$ & $\begin{array}{c}59 \\
(63,4) \\
\end{array}$ \\
\hline Valor de p* & \multicolumn{3}{|c|}{0,207} & \multicolumn{3}{|c|}{0,888} \\
\hline \multicolumn{7}{|l|}{ LDL-c** $^{* *}$} \\
\hline $\begin{array}{c}\text { Ótimo } \\
(<100 \mathrm{mg} / \mathrm{dL})\end{array}$ & $\begin{array}{c}12 \\
(17,4) \\
\end{array}$ & $\begin{array}{c}18 \\
(26,1) \\
\end{array}$ & $\begin{array}{c}39 \\
(56,5) \\
\end{array}$ & $\begin{array}{c}33 \\
(25,4) \\
\end{array}$ & $\begin{array}{c}41 \\
(31,5) \\
\end{array}$ & $\begin{array}{c}56 \\
(43,1) \\
\end{array}$ \\
\hline $\begin{array}{c}\text { Desejável } \\
(100-129 \mathrm{mg} / \mathrm{dL})\end{array}$ & $\begin{array}{c}13 \\
(11,9)\end{array}$ & $\begin{array}{c}25 \\
(22,9)\end{array}$ & $\begin{array}{c}71 \\
(65,2)\end{array}$ & $\begin{array}{l}31 \\
(17)\end{array}$ & $\begin{array}{c}32 \\
(17,6)\end{array}$ & $\begin{array}{c}119 \\
(65,4)\end{array}$ \\
\hline $\begin{array}{c}\text { Limítrofe } \\
(130-159 \mathrm{mg} / \mathrm{dL})\end{array}$ & $\begin{array}{c}5 \\
(4,1) \\
\end{array}$ & $\begin{array}{c}25 \\
(20,7) \\
\end{array}$ & $\begin{array}{c}91 \\
(75,2)\end{array}$ & $\begin{array}{c}12 \\
(7,9) \\
\end{array}$ & $\begin{array}{c}22 \\
(14,6) \\
\end{array}$ & $\begin{array}{c}117 \\
(77,5) \\
\end{array}$ \\
\hline $\begin{array}{c}\text { Alto } \\
(\geq 160 \mathrm{mg} / \mathrm{dL})\end{array}$ & $\begin{array}{c}4 \\
(5,9)\end{array}$ & $\begin{array}{c}9 \\
(13,2)\end{array}$ & $\begin{array}{c}55 \\
(80,9)\end{array}$ & $\begin{array}{c}5 \\
(6,2)\end{array}$ & $\begin{array}{c}12 \\
(14,8)\end{array}$ & $\begin{array}{c}64 \\
(79)\end{array}$ \\
\hline Valor de p* & \multicolumn{3}{|c|}{0,010} & \multicolumn{3}{|c|}{$\leq 0,001$} \\
\hline
\end{tabular}

*Valores estatisticamente significantes com $\mathrm{p}<0,05 .{ }^{* *}$ Teste Exato de Fisher

Os resultados da classificação das dislipidemias de indivíduos que apresentaram alteração nas frações lipídicas ( $n=737$ ), segundo o gênero e faixa etária estão descritos na tabela 4. Observou-se um percentual mais elevado de hipercolesterolemia, hipertrigliceridemia isolada, hiperlipidemia mista e HDL-c baixo na faixa etária a partir de 40 anos de idade, em ambos os sexos.

Tabela 4 - Classificação das dislipidemias de acordo com os critérios adotados pela $\vee$ Diretriz Brasileira de Prevenção da Aterosclerose $(n=737)$.

\begin{tabular}{c|c|c|c|c|c|c}
\hline \multirow{2}{*}{$\begin{array}{c}\text { Classificação das } \\
\text { Dislipidemias }\end{array}$} & \multicolumn{5}{|c|}{ Masculino } & \multicolumn{3}{c}{ Feminino etária } \\
\cline { 2 - 7 } & $20-29$ & $30-39$ & $\geq 40$ & $20-29$ & $30-39$ & $\geq 40$ \\
\hline $\begin{array}{c}\text { Hipercolesterolemia } \\
\text { Isolada }\end{array}$ & $\begin{array}{c}4 \\
(4,3 \%)\end{array}$ & $\begin{array}{c}12 \\
(12,8 \%)\end{array}$ & $\begin{array}{c}78 \\
(82,9 \%)\end{array}$ & $\begin{array}{c}5 \\
(5,7 \%)\end{array}$ & $\begin{array}{c}13 \\
(14,8 \%)\end{array}$ & $\begin{array}{c}70 \\
(79,5 \%)\end{array}$ \\
\hline $\begin{array}{c}\text { Hipertrigliceridemia } \\
\text { Isolada }\end{array}$ & 9 & 29 & 143 & 17 & 29 & 124 \\
$(5 \%)$ & $(16 \%)$ & $(79 \%)$ & $(10 \%)$ & $(17,1 \%)$ & $(72,9 \%)$ \\
\hline $\begin{array}{c}\text { Hiperlipidemia } \\
\text { Mista }\end{array}$ & 2 & $\begin{array}{c}6 \\
(3,3 \%)\end{array}$ & $\begin{array}{c}53 \\
(9,8 \%)\end{array}$ & $\begin{array}{c}1 \\
(86,9 \%)\end{array}$ & $\begin{array}{c}(2,5 \%) \\
(10 \%)\end{array}$ & $\begin{array}{c}35 \\
(87,5 \%)\end{array}$ \\
\hline HDL-C baixo & 15 & 30 & 95 & 29 & 56 & 149 \\
$(10,7 \%)$ & $(21,4 \%)$ & $(67,9 \%)$ & $(12,4 \%)$ & $(23,9 \%)$ & $(63,7 \%)$ \\
\hline
\end{tabular}




\section{Discussão}

Relevantes estudos avaliando as dislipidemias em adultos são encontrados na literatura, no entanto, apresentam diferentes parâmetros de classificação e amostragem populacional. Neste estudo, foi adotado como referencial os critérios da V Diretriz Brasileira de Dislipidemia e Prevenção da Aterosclerose ${ }^{5}$.

O percentual das doenças cardiovasculares (DVC) tem aumentado progressivamente, inicialmente nos países desenvolvidos e mais recentemente naqueles em vias de desenvolvimento, por influência da modificação do estilo de vida e o aumento do peso da população ${ }^{10,11}$.

A prevalência de alteração em componentes do perfil lipídico encontrada $(78,1 \%)$ foi superior ao estudo realizado em Goiânia $(\mathrm{GO})^{12}$, no qual observaram um percentual de $51,2 \%$. Isso pode ser explicado pelo estilo de vida, genética, alimentação, idade e sexo da população ${ }^{5}$.

Foi observada uma diferença significativa nos valores de HDL-centre os sexos. Homens apresentaram maior percentual $(60,1 \%)$ de valores baixos de HDL-c (<40mg/dL). Em contrapartida, as mulheres apresentaram percentuais maiores na faixa desejável, na qual os níveis são superiores a $60 \mathrm{mg} / \mathrm{dL}$. O percentual encontrado foi superior aos observados em Estudo Longitudinal de Saúde do Adulto (ELSA-Brasil) ${ }^{8}$ e em Goiânia (GO) ${ }^{12}$, onde os pesquisadores relataram níveis baixos de HDL-c em $37,3 \%$ e $41,44 \%$ dos homens, respectivamente. Níveis baixos de HDL-c aumentam os riscos de desenvolvimento de doenças cardiovasculares, pois essa lipoproteína exerce a função de proteção contra a aterogênese ${ }^{5}$.

$\mathrm{O}$ aumento dos triglicerídeos é um distúrbio lipídico relativamente frequente em uma população, exercendo um papel primário na formação de aterosclerose e predisposição à doença cardiovascular ${ }^{13}$. Neste estudo, verificou-se níveis de triglicerídeos mais elevados nos homens em relação às mulheres, sendo esta associação estatisticamente significante. Estes dados corroboram com os valores encontrados em um estudo realizado no interior de Minas Gerais ${ }^{14}$.

O principal fator de risco não modificável para a DVC é a idade. A partir da terceira década de vida, as lesões do endotélio vascular tornam-se mais evidentes e as suas consequências clínicas surgem por volta dos 40 anos ${ }^{15}$. Observou-se uma diferença estatisticamente significativa da idade sobre os níveis lipídicos de colesterol total e LDL-c. Em ambos os sexos, o colesterol foi mais elevado nos indivíduos de maior faixa etária (acima dos 40 anos de idade). A variação observada do colesterol total com a idade é semelhante com as observações encontradas em outros estudos ${ }^{10,16}$.

Em relação ao LDL-c, a idade também apresentou diferença significativa quando associada ao sexo masculino e feminino. Quanto maior a idade, mais elevados foram os níveis de LDL-c. Essa lipoproteína de baixa densidade possui uma relação direta no surgimento de DVC com elevados níveis de LDL-C, uma vez que estas partículas participam do desenvolvimento das placas ateroscleróticas ${ }^{5,17}$.

As dislipidemias apresentaram um maior percentual em indivíduos mais velhos e de ambos os sexos. Estes resultados podem ser explicados pelo estilo de vida e comportamento adotados pelos indivíduos dessa faixa etária; como tabagismo, sedentarismo, etilismo e hábitos alimentares ${ }^{8}$. Os homens com idade mais avançada tendem a acumular maior teor de gordura visceral do que as mulheres, sendo um importante fator de risco associado a outros fatores modificáveis para o desenvolvimento das dislipidemias e aparecimento de DVC ${ }^{18}$. Nas mulheres, outro fator que pode ser determinante na elevação dos lipídeos é a redução de estrógenos ${ }^{19}$. Estudos relataram que os níveis séricos de colesterol total, triglicerídeos e LDL-c podem aumentar da pré-menopausa para a pós-menopausa ${ }^{20} \mathrm{e}$ esse aumento pode estar relacionado com as alterações no índice de massa corporal e na composição corporal ocorridas com o advento do climatério e do envelhecimento ${ }^{21}$.

Dentre as limitações deste estudo estão o delineamento transversal que não permite a inferência de causa e efeito, e da amostra ser representativa apenas do centro em que foram coletados os dados. Entretanto, é o primeiro trabalho desenvolvido na região, apresentando as estimativas de prevalência do perfil lipídico de adultos com a determinação das frequências por sexo e idade com referencial padronizado.

\section{Considerações Finais}

A prevalência de alterações no perfil lipídico foi elevada entre os indivíduos a partir de 40 anos de idade, o que demonstra uma forte tendência ao desenvolvimento de doenças cardiovasculares. 0 conhecimento de prevalências é fundamental para que políticas públicas de saúde em âmbito local sejam desenvolvidas. Os dados encontrados 
contribuirão para a melhor caracterização epidemiológica do risco cardiovascular na população estudada, permitindo um adequado planejamento de estratégias preventivas de saúde pública para a região. Ademais, mais trabalhos devem ser desenvolvidos na região a fim de ampliarem o conhecimento acerca do perfil lipídico desta população de traços e hábitos culturais característicos da fronteira do Rio Grande do Sul, sul do Brasil.

\section{Referências}

1. Mansur, A.P.; Favarato, D. Mortalidade por doenças cardiovasculares no Brasil e na região metropolitana de São Paulo: atualização 2011. Arq. Bras. Cardiol., São Paulo, v. 99, n. 2, p. 755-761, Aug. 2012

2. Salvaro RP, Avila Júnior S. Perfil lipídico e a sua relação com fatores de risco cardiovascular em estudantes de nutrição. Rev SOCERJ. 2009; 22(5): 309-17.

3. Trapé, A.; Lizzi, E.; Jacomini, A.; Hott, S.; Bueno, C.R.; Zago, A.S. Aptidão física e nível habitual de atividade física associados à saúde cardiovascular em adultos e idosos. Medicina (Ribeirão Preto) 2015;48(5): 457-66

4. Pereira, R. A relação entre dislipidemia e diabetes mellitus tipo 2. Cad. UniFOA. 2011; 17 (2): 101-6.

5. Xavier, H.T.; Izar, M.C.; Faria Neto, JR.; Assad, M.H.; Rocha, V.Z.; Sposito, A.C. et al. V Diretriz Brasileira de Dislipidemias e Prevenção da Aterosclerose.Arq Bras Cardiol. 2013; 101(4): 1-20.

6. Siqueira, A.F.A.; Abdalla, D.S.P.; Ferreira, S.R.G. LDL: da síndrome metabólica à instabilização da placa aterosclerótica. Arq Bras Endocrinol Metab. 2006; 50(2): 334-43.

7. Pereira, P.B.; Arruda, I.K.G.; Cavalcanti, A.M.T.S.; Diniz, A.S. Perfil lipídico em escolares de Recife - PE. Arq Bras Cardiol. 2010; 95(5): 606-13.

8. Silva, R.C.; Diniz, M.F.H.S; Alvim, S.; Vidigal, P.G.; Fedeli, L.M.G.; Barreto, S.M. Atividade Física e Perfil Lipídico no Estudo Longitudinal de Saúde do Adulto (ELSA-Brasil). Arq Bras Cardiol. 2016; 107(1):10-19.

9. Friedewald, W.T.; Levi, R.I.; Fredrickson, D.S. Estimation of the concentration of low density lipoproteins cholesterol in plasma without use of the ultracentrifuge. Clin Chem. 1972; 18(6): 499-502.

10. Cortez-Dias, N.; Martins, S.R.; Belo, A.; Fiúza, M. Caracterização do perfil lipídico nos utentes dos cuidados de saúde primários em Portugal. Rev Port Cardiol. 2013; 32 (12): 987-96.

11. Alakkas, Z.; Alswat, K.A.; Otaibi, M.A.; Althobaiti, T.; Alzaidi, N.; Khalek, E.A.; Alfif, A. The prevalence and the clinical characteristics of metabolic syndrome patients admitted to the cardiac care unit. J Saudi Heart Assoc 2016; 28:136-143.

12. Montel, M.G.; Costa, S.H.N.; Blanch, G.T. Prevalência de dislipidemia em pacientes atendidos em hospital militar de Goiânia-Goiás. Estudos, Goiânia. 2014; 41(4): 715-20.

13. Toth, P.P. Triglyceride-rich lipoproteins as a causal factor for cardiovascular disease. Vascular Health and Risk Management 2016:12 pag. 171-183

14. Pimenta, A.M.; Kac, G.; Gazzinelli, A.; Corrêa-Oliveira, R.; Velásquez-Meléndez, G. Associação entre obesidade central, triglicerídeos e hipertensão arterial em uma área rural do Brasil. Arq Bras Cardiol. 2008; 90(6): 419-25.

15. Sözmen, K.; Ünal, B.; Sakarya, S.; Dinç, G.; Yardım, N.; Keskinkılıç, B.; Ergör, G. Determinants of prevalence, awareness, treatment and control of high LDL-C in Turkey. Anatol J Cardiol 2016; 16: 370-84

16. Hoffmann, K.; Matyiaszczyk, P.; Zaporowska-StachoWiak, I.; Kostrzewska, M.; Piorunek, T.; Minczykowskp, A.; Bryl, W. Lipid profile of polish primary care patients aged 35-55 years living in the district of pleszew. Acta Pol Pharm. 2016 Mar-Apr;73(2):537-46.

17. Ahmed, H.M.; Miller, M.; Nasir, K.; McEvoy, J.W.; Herrington, D.; Blumenthal, R.S.; Blaha, M.J. Primary Low Level of High-Density Lipoprotein Cholesterol and Risks of Coronary Heart Disease, Cardiovascular Disease, and Death: Results From the Multi-Ethnic Study of Atherosclerosis.

18. Santos, C.M.; Silva, C.S.; Araujo, E.C.; Arruda, I.K.G.; Diniz, A.S.; Cabral, P.C. Perfil lipídico e glicídico de pacientes atendidos em ambulatório e sua correlação com índices antropométricos. Rev Port Cardiol. 2013; 32(1): 35-41.

19. Graff-Iversen, S; Thelle, D.S.; Hammar, N. Serum lipids, blood pressure and body weight around the age of the menopause. Eur J Cardiovasc Prev Rehabil. 2008; 15 (1): 83-88.

20. Stachowiak, G.; Pertyński, T.; Pertyńska-Marczewska, M. Metabolic disorders in menopause. Prz Menopauzalny 2015; 14(1): 59-64

21. Meirelles, R.M.R. Menopausa e síndrome metabólica. Arq Bras Endocrinol Metab. 2014;58/2. 


\section{Carolina Simões Pires Ribeiro}

Endereço para correspondência - Gov. Agamenon Magalhães, $n^{\circ} 173$,

CEP: 80210-170, Curitiba, PR, Brasil.

E-mail: carolinaspribeiro@gmail.com

Lattes: http://lattes.cnpq.br/9121842164308562

Beatris Gonzalez Cademartori - biacademartori@yahoo.com.br

Andréa da Silva Ramos Rocha - ararocha@terra.com.br

Enviado em 05 de abril de 2016.

Aceito em 06 de agosto de 2016. 
